\title{
Primary Spinal Cord Melanoma - An Uncommon Entity
}

\author{
Ritodhi Chatterjee, Fábio A. Nascimento (1), Kent A. Heck, Alexander E. Ropper, \\ Anita L. Sabichi
}

Keywords: Primary spinal cord melanoma, Melanoma, Spinal cord tumor, Intramedullary tumor

doi:10.1017/cjn.2019.28

Can J Neurol Sci. 2019; 46: 348-350

We report a 78-year-old white male with a history of nonmelanoma skin cancer who presented with a 2-month history of fatigue, worsening right-sided weakness and paresthesia, and urinary incontinence. Examination revealed weakness in the right hand and lower extremity (MRC 0/5), decreased sensation in the latter, and a T4 sensory level. Reflexes were absent in the right lower extremity, Babinski sign was present on the left, and rectal tone was absent.

Neuroimaging showed a contrast-enhancing C7 intramedullary lesion with associated hemorrhage and surrounding edema (Figure 1). Preliminary differential diagnoses included metastatic versus primary spinal cord melanoma, melanocytoma, intermediate-grade melanocytic tumor, melanotic schwannoma, and common intramedullary tumors such as ependymoma and astrocytoma. The patient underwent an intradural tumor resection via C6-T1 laminectomy. Intraoperatively, the lesion was soft and black with an intratumoral hematoma. Histologic examination revealed diffuse proliferation of poorly nested epithelioid cells with severe anisonucleosis, prominent nucleoli, and occasional mitoses (Figure 2). Additional work-up with whole-body positron emission tomography/computed tomography (PET/CT), skin/ocular examinations, and upper/lower GI endoscopies were unremarkable, leading to diagnosis of primary spinal cord melanoma. He did not receive adjuvant radiation or systemic therapy. At 18-month follow-up, he has remained without any clinical evidence of disease.

Primary CNS melanocytic neoplasms such as melanotic schwannoma, meningeal melanocytoma, and blue nevus of the CNS comprise $1 \%$ of all melanoma cases. ${ }^{1}$ Primary spinal cord melanomas are even rarer; to our knowledge, fewer than 70 cases have been reported. Most spinal melanomas are metastatic from the skin, uvea, or vaginal or gastrointestinal mucosa; primary lesions can originate in the meninges or spinal parenchyma. $^{2}$ They occur most often in the fifth decade of life and appear most commonly in the thoracic region of the spinal cord. ${ }^{3}$ A presenting symptom is typically back or neck pain accompanied by progressive, asymmetric myelopathy. Diagnosis requires absence of other CNS tumors, lack of extra-CNS lesions, and pathological confirmation. ${ }^{4}$ Histologically, melanoma is characterized by large, pleomorphic epithelioid or spindled cells with irregular nuclei, necrosis, and high mitotic index. Positive staining for HMB-45 and S100 protein is supportive, and markers such as vimentin, neuroendocrine markers, or EMA can help rule out other pigmented tumors. ${ }^{5}$ MRI imaging features T1 hyperintensity and T2 iso/hypointensity with subtle contrast enhancement. ${ }^{1}$

There is little data on management and prognosis of primary spinal cord melanoma. Generally, surgical treatment with resection is elected. The benefits of adjuvant chemo and/or radiotherapy are unclear. Some studies credit a multidisciplinary approach involving chemo and radiotherapy, ${ }^{2}$ whereas others demonstrate no survival benefit, but possible decrease in risk of metastasis. ${ }^{6}$ Survival outcomes vary dramatically from 3 months to 21 years, and late recurrences have been reported. ${ }^{7}$ According to a retrospective study, the 12-month survival was $89.6 \%$ and the 72 -month survival was $39.6 \% .^{6}$

This report underlines the importance of a comprehensive diagnostic approach involving imaging with spine MRI, wholebody CT and PET, detailed histopathological examination, and thorough gastrointestinal, ophthalmologic, dermatologic, and gynecologic evaluation to determine whether the melanoma is metastatic to the spine or primary spinal melanoma. This distinction is of paramount importance as primary lesions are associated with better outcomes following early surgical intervention and, controversially, adjuvant chemoradiotherapy.

\section{Disclosure}

AER reports personal fees from Globus Medical, Inc. and Stryker Corporation outside the submitted work. All other authors report no disclosures.

From the Department of Neurology, Baylor College of Medicine, Houston, Texas, USA (RC, FAN); Department of Pathology, Baylor College of Medicine, Houston, Texas, USA (KAH); Department of Neurosurgery, Baylor College of Medicine, Houston, Texas, USA (AER); Section of Hematology and Oncology, Department of Medicine, Baylor College of Medicine, Houston, Texas, USA (ALS)

Received December 30, 2018. Final Revisions Submitted February 26, 2019. Date of Acceptance February 28, 2019.

Correspondence to: Fábio A. Nascimento, Department of Neurology, Baylor College of Medicine (BCM), 1 Baylor Plaza, Houston, Texas 77030, USA. Email: Nascimento. Fabio.A@gmail.com; Fabio. Nascimento@bcm.edu 


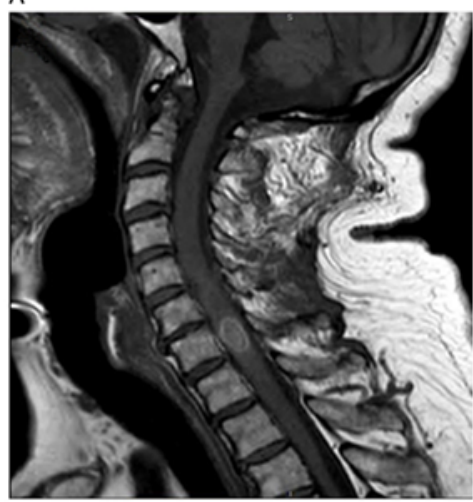

D

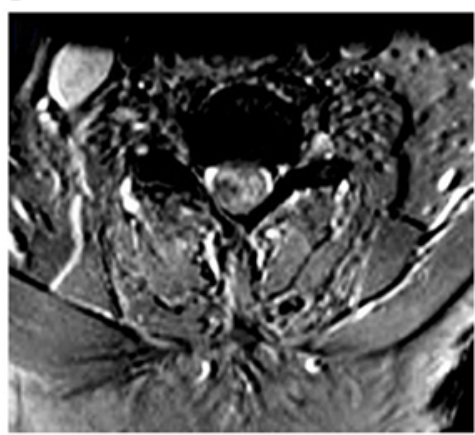

B

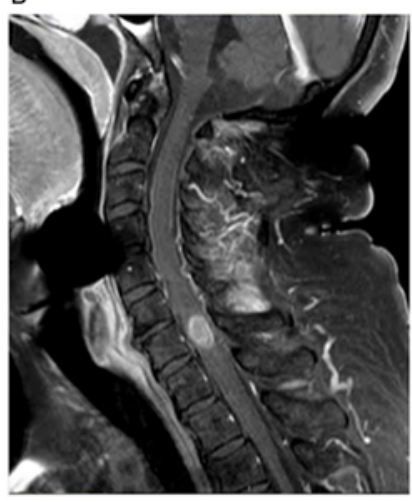

$\mathrm{E}$



C



$\mathrm{F}$

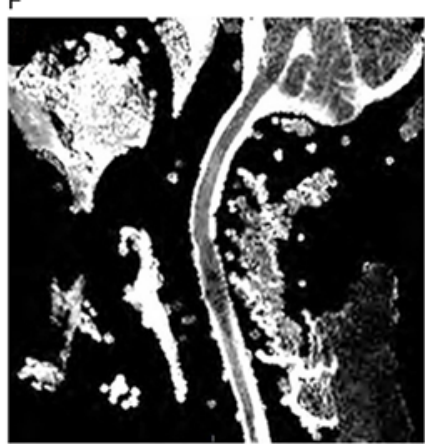

Figure 1: Spine MRI. Sagittal T1 (A), post-contrast sagittal T1 (B), sagittal T2 (C), and axial T2-FFE (D) show an enhancing intramedullary lesion located at the $C 7$ level associated with cord hemorrhage and surrounding nonenhancing signal changes extending from C3 to T3. Sagittal DWI $(E)$ and sagittal ADC $(F)$ reveal intralesional diffusion restriction suggestive of hypercellularity.

A

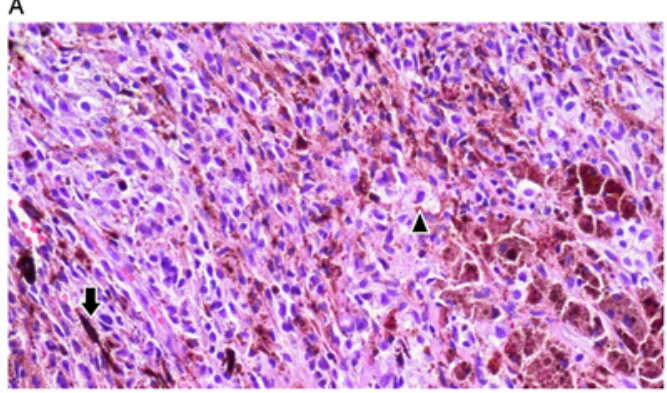
C

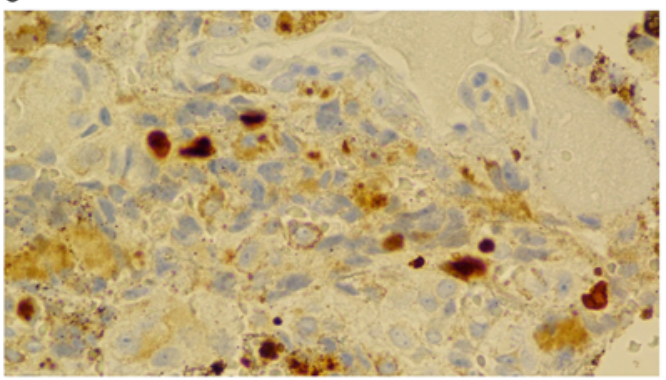

B

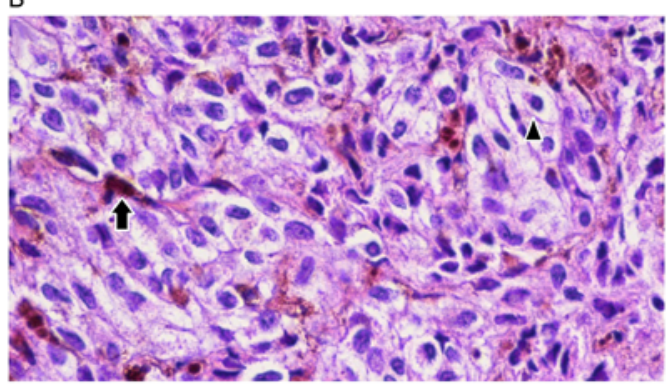

$\mathrm{D}$

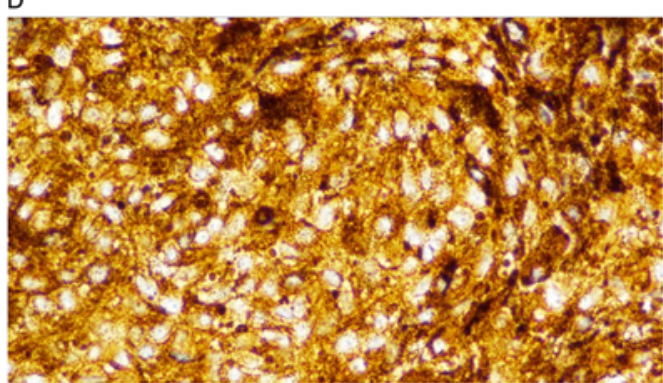

Figure 2: Variable histology of the lesion. (A and B) Hematoxylin and eosin stain (50X and 100X magnification, respectively) showing neoplastic epithelioid cells (arrowheads) with frequent melanin pigmentation (full arrows). (C) Ki-67 immunoperoxidase technique confirms a proliferation index of 10\% or more in the tumor (nuclear immunopositivity), strongly supporting diagnosis of melanoma over melanocytoma. (D) Tumor cells expressing $H M B-45$, a cytoplasmic melanoma marker (immunoperoxidase, DAB). 


\section{STATEMENT OF AUTHORShIP}

RC: chart and literature review, and drafting manuscript.

FAN: content design, drafting, and revising manuscript.

$\mathrm{KAH}$ : case report supervision and revising manuscript.

AER: case report supervision and revising manuscript.

ALS: case report supervision and revising manuscript.

\section{REFERENCES}

1. Farrokh D, Fransen P, Faverly D. MR findings of a primary intramedullary malignant melanoma: case report and literature review. AJNR Am J Neuroradiol. 2001;22(10):1864-6.

2. Ganiüsmen O, Özer FD, Mete M, Özdemir N, Bayol Ü. Slow progression and benign course of a primary malign melanoma of a lumbar nerve root. Clin Neurol Neurosurg. 2012; 114(2):166-8.

3. Kim MS, Yoon DH, Shin DA. Primary spinal cord Melanoma. J Korean Neurosurg Soc. 2010;48(2):157-61.

4. Hayward RD. Malignant melanoma and the central nervous system: a guide for classification based on the clinical findings. J Neurol Neurosurg Psychiatry. 1976;39(6):526-30.

5. Ruelle A, Tunesi G, Andrioli G. Spinal meningeal melanocytoma: case report and analysis of diagnostic criteria. Neurosurg Rev. 1996;19(1):39-42.

6. Zhang M, Liu R, Xiang Y, et al. Primary spinal cord melanoma: a case report and a systemic review of overall survival. World Neurosurg. 2018;114:408-20.

7. Fuld AD, Speck ME, Harris BT, et al. Primary melanoma of the spinal cord: a case report, molecular footprint, and review of the literature. J Clin Oncol. 2011;29(17):499-502. 